\title{
Black hole bound on the number of species and quantum gravity at CERN LHC
}

\author{
Gia Dvali ${ }^{1,2}$ and Michele Redi ${ }^{2,3}$ \\ ${ }^{1}$ CERN, Theory Division, CH-1211 Geneva 23, Switzerland \\ ${ }^{2}$ CCPP, Department of Physics, New York University, 4 Washington Place, New York, New York 10003, USA \\ ${ }^{3}$ ITPP, EPFL, CH-1015, Lausanne, Switzerland
}

(Received 9 November 2007; published 21 February 2008)

\begin{abstract}
In theories with a large number $N$ of particle species, black hole physics imposes an upper bound on the mass of the species equal to $M_{\text {Planck }} / \sqrt{N}$. This bound suggests a novel solution to the hierarchy problem in which there are $N \approx 10^{32}$ gravitationally coupled species, for example $10^{32}$ copies of the standard model. The black hole bound forces them to be at the weak scale, hence providing a stable hierarchy. We present various arguments, that in such theories the effective gravitational cutoff is reduced to $\Lambda_{G} \approx M_{\text {Planck }} / \sqrt{N}$ and a new description is needed around this scale. In particular, black holes smaller than $\Lambda_{G}^{-1}$ are already no longer semiclassical. The nature of the completion is model dependent. One natural possibility is that $\Lambda_{G}$ is the quantum gravity scale. We provide evidence that within this type of scenarios, contrary to the standard intuition, micro-black-holes have a (slowly fading) memory of the species of origin. Consequently, the black holes produced at LHC will predominantly decay into the standard model particles, and negligibly into the other species.
\end{abstract}

DOI: 10.1103/PhysRevD.77.045027

PACS numbers: 12.10.Dm, 04.70.Dy, 12.60.Cn

\section{INTRODUCTION}

It is conceivable that the mysterious radiative stability of the hierarchy between the Planck and the weak scales is imposed by the intrinsic consistency of the underlying gravitational dynamics. If so, this underlying principle should manifest itself as a consistency relation between the different scales expressed in terms of radiatively insensitive quantities of the theory, such as topological numbers, representations of the symmetry groups, or simply the number of species.

An example of such a consistency bound was proved recently in [1]. It was shown that, in theories with a large number $N$ of species with a mass scale $\Lambda$, black hole (BH) physics imposes a relation between the Planck scale $\left(M_{P}\right)$ and the mass of the species $(\Lambda)$,

$$
M_{P}^{2} \geq N \Lambda^{2},
$$

up to a factor that scales as $\approx \log N$ at large $N$. In other words, the large number of species weakens gravity by $1 / N$.

Then, the following simple solution to the hierarchy problem emerges. Stability of the weak scale can be explained by the fact that there are $N \approx 10^{32}$ species beyond the standard model (SM). For instance, these new species could be $N$ copies of the SM, perhaps related by permutation symmetry, and only gravitationally coupled to each other, as in Fig. 1.

At first sight, from a perturbative point of view it might seem mysterious what guarantees the stability of the mass of the particle species. In the present work we provide various arguments that the $N$-species effective theory breaks down at or below the reduced scale,

$$
\Lambda_{G} \approx M_{P} / \sqrt{N} \text {. }
$$

That is, when the bound (1.1) is saturated, $\Lambda_{G}$ approaches the mass $\Lambda$ of the $N$ species and a completion is needed right above that scale. From this point of view, the fundamental scale of the theory is $\Lambda_{G}$ while $M_{P}$ is a derived quantity whose large value is due to the large multiplicity of species.

One strong indication that new gravitational dynamics should appear around the scale $\Lambda_{G}$ comes from the fact that the BHs of size $\Lambda_{G}^{-1}$, much larger than Planck length, have lifetime $\tau_{\mathrm{BH}} \approx \Lambda_{G}^{-1}$ so they already probe the microscopic theory of gravity. The reduced cutoff (1.2) in particular reconciles the bound (1.1) with naturalness arguments since in the absence of tuning the perturbative renormalization of the Planck mass due to the $N$ species with cutoff $\Lambda_{G}$ saturates (1.2). This idea was used in [2] as a possible origin of the large value of $M_{P}$. This also agrees with perturbative arguments by Veneziano [3].

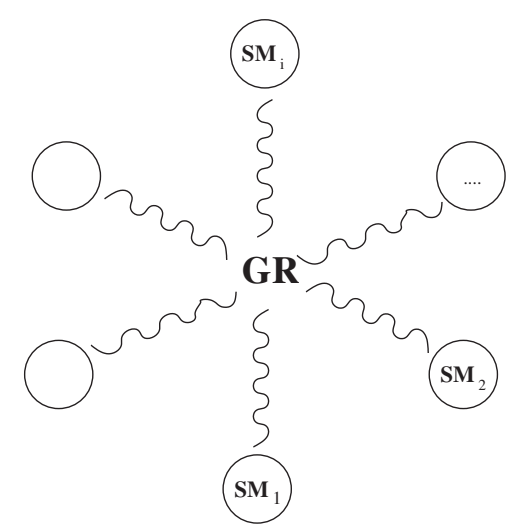

FIG. 1. We consider as a possible solution of the hierarchy problem the existence of particle species interacting gravitationally. 
The nature of the cutoff $\Lambda_{G}$ is not uniquely determined by the consistency of the low energy theory. One natural possibility that we investigate is that gravity becomes strong and $\Lambda_{G}$ is the quantum gravity scale. This is in fact the case in the large extra-dimension scenario [4] which can be understood as a special case of our large $N$ theories. We focus on single-cutoff scenarios, in which $\Lambda_{G}$ sets a universal cutoff both for gravity as well as for the other particle species (SM and its copies). The most spectacular signature of this type of models is that SM collisions at energies of order $\mathrm{TeV}$ will produce micro-BHs and the LHC will directly probe the quantum gravity regime. The micro-BHs will have very peculiar features. As we will show, contrary to the naive intuition, BHs produced in particle collisions at energy $\Lambda_{G}$ have a slowly fadingaway memory of the particular copy of the species that produced them. As a result, the BHs produced at the LHC in the collision of SM particles will predominantly decay into our SM particles and only with a strongly suppressed probability into the other copies.

We propose a dynamical interpretation to this fadingaway memory in terms of slowly decaying quantum or classical hair of the black holes. This hair is not associated with any long range classical field. It either could be a hair under some discrete symmetry, or under some massive gauge fields that are associated with the given copy of species. This slowly decaying $\mathrm{BH}$ hair then creates a slowly fading-away memory of the site of origin in the space of species. This implies that there is some notion of locality in the space of species, although the only perturbative link between the copies is the universally coupled graviton, and the label of the copies is not necessarily a coordinate in any real space.

This paper is organized as follows. In Sec. II we review the argument determining the bound on the Planck scale. In Sec. III we discuss perturbative and nonperturbative arguments showing that some new gravitational dynamics must appear below the scale $\Lambda_{G}$. Section IV contains examples of theories where the bound on $M_{P}$ is saturated. Finally in Sec. V we consider the special properties of black holes in the theories with quantum gravity scale $\Lambda_{G}$.

\section{THE BLACK HOLE BOUND ON THE NUMBER OF SPECIES}

We shall first briefly reproduce the main idea behind the proof of the bound of [1].

The proof is the simplest when each sector of species has an exactly conserved quantum number. Consider $N$ species of the quantum fields $\Phi_{j}, j=1,2, \ldots N$, of mass $\Lambda$, each carrying a separately conserved $Z_{2}$-charge. The system is invariant under an exact discrete $Z_{2}^{N} \equiv Z_{2}^{(1)} \times Z_{2}^{(2)} \times$ $\cdots Z_{2}^{(N)}$ symmetry, under the independent sign flips of the fields.

In order to prove the relation (1.1), we can perform the following thought experiment. Let us prepare a macro- scopic BH carrying the maximal $N$-units of the discrete charge. This can be obtained by taking a large neutral $\mathrm{BH}$ and throwing one particle from each species into it. The BH prepared in such a way will carry exactly $N$-units of the conserved discrete charge. To avoid entering the discussions on the black hole information loss issues, it is useful to consider these $Z_{2} \mathrm{~s}$ as the gauged discrete symmetries. As shown by Krauss and Wilczek [5], the information about the absorbed charge then can be monitored by the Aharonov-Bohm effect at infinity, using for example $Z_{2}$-cosmic strings and cannot be lost.

Because of the charge conservation, the information about the $Z_{2}^{N}$-charge hosted by the black hole must be revealed after its evaporation. For a black hole with Hawking temperature $T_{H}$, the probability of the emission of a heavy particle of mass $\Lambda \gg T_{H}$ is exponentially suppressed by a Boltzmann factor $\approx \mathrm{e}^{-\left(\Lambda / T_{H}\right)}$. Thus, our black hole with $N$ units of the $Z_{2}^{N}$-charge can start emitting particles from the $N$ species, only after its temperature drops to $T_{H} \approx \Lambda$. At this point, the mass of the black hole is $M_{\mathrm{BH}}^{*} \approx \frac{M_{P}^{2}}{\Lambda}$. Starting from this moment, the black hole can start revealing back the stored charge, in the form of the $N$ particles species. However, by conservation of energy, the maximum number of particles that can be emitted by the black hole is

$$
n_{\max } \approx \frac{M_{P}^{2}}{\Lambda^{2}} .
$$

These states should carry the same $Z_{2}^{N}$-charge as the original $N$-particles. Thus, $n_{\max }=N$, which proves the equation (1.1).

In other words, the key point of the proof is that the amount of the maximal discrete charge which is stored in the initial black hole scales as $N$, but the temperature at which the black hole starts giving back this charge essentially does not scale with $N$. Hence, the only way to avoid inconsistency is to scale the Planck mass ${ }^{2}$ as $N$. With minor assumption the same conclusion holds generically in the presence of $N$ species.

\section{THE QUANTUM GRAVITY SCALE}

The question that we wish to answer now is what is the cutoff of the theory. We present several arguments that in the presence of $N$ species new gravitational dynamics must appear below the scale $\Lambda_{G} \approx M_{P} / \sqrt{N}$. While this holds in general, when the BH bound (1.1) is saturated it implies that the theory must break down right above the highest mass scale of the species.

\section{A. Black hole argument}

A strong nonperturbative argument supporting the claim that a gravitational cutoff should be at the scale $\Lambda_{G}$ is provided by the fact that the lifetime of the $\mathrm{BHs}$ of size $\Lambda_{G}^{-1}$ is $\approx \Lambda_{G}^{-1}$. 
To see this, consider a BH with temperature $T_{H} \approx \Lambda_{G}$. Let us assume first that the $\mathrm{BH}$ does not carry the conserved charge of species number. In semiclassical approximation the decay rate of the $\mathrm{BH}$ is

$$
\frac{d M_{\mathrm{BH}}}{d t} \approx-N T_{H}^{2} .
$$

Using the relation between the black hole mass and the temperature $T_{H} \approx M_{P}^{2} / M_{\mathrm{BH}}$, we can reexpress this as

$$
\tau_{\mathrm{BH}} \approx \frac{1}{N} \int_{0}^{M_{i n}} \frac{M_{\mathrm{BH}}^{2}}{M_{P}^{4}} d M_{\mathrm{BH}},
$$

where $M_{\text {in }}$ is the initial mass, which for the black hole of size $\Lambda_{G}^{-1}$ is $M_{i n} \approx \frac{M_{P}^{2}}{\Lambda_{G}}$. This gives

$$
\tau_{\mathrm{BH}} \approx \frac{M_{B H}^{3}}{N M_{P}^{4}} \approx \frac{1}{\Lambda_{G}} \text {. }
$$

If the $\mathrm{BH}$ has $N$ units of the conserved charge, this calculation has to be slightly modified to take into account the fact that due to the conservation of charge the number of species available for emission decreases as the $\mathrm{BH}$ evaporates. In this case,

$$
\frac{d M_{\mathrm{BH}}}{d t} \approx-n\left(M_{\mathrm{BH}}\right) T_{H}^{2},
$$

where $n\left(M_{\mathrm{BH}}\right)=\frac{M_{\mathrm{BH}}}{\Lambda_{G}}$ is the number of available species. Going through the same steps as above, one finds that the lifetime of the $\mathrm{BH}$ is again given by (3.3).

The fact that the lifetime becomes comparable to the size of the $\mathrm{BH}$ implies that they cannot be treated as semiclassical four-dimensional $\mathrm{BHs}$ with well-defined Hawking temperature, since the temperature $T_{H}$ itself changes on the time scale $\approx T_{H}^{-1}$. Therefore we conclude that $\Lambda_{G}$ determines the critical scale beyond which new gravitational dynamics must appear.

\section{B. Perturbative arguments}

We now turn to perturbative arguments (see [3] for related work). To simplify the discussion let us assume that there are $N$ species with mass $\Lambda \lesssim \Lambda_{G}$ which are weakly coupled. It is easy to see that the perturbation theory breaks down at the scale $M_{P} / \sqrt{N}$. Consider the correction to the graviton propagator due to the loops of the $N$ particles. Neglecting the index structure, this is given by

$$
\frac{1}{M_{P}^{2}} \frac{1}{p^{2}}\langle T(p) T(-p)\rangle \frac{1}{p^{2}},
$$

where $T$ denotes the energy momentum tensor. The two point function of $T$ has UV sensitive contribution and a nonlocal, calculable piece associated to the production of the particles. The form of the correction can be obtained by noting that well above the mass of the particles the theory becomes approximately conformal. By conformal invari- ance one has

$$
\langle T(p) T(-p)\rangle=c p^{4} \log \frac{p^{2}}{\Lambda^{2}},
$$

where the constant $c$ is the central charge counting the number of degrees of freedom of the theory, i.e. $c \approx N$. Comparing this with the tree level result, one finds that the perturbative expansion fails at the scale

$$
p^{*} \approx \frac{M_{P}}{\sqrt{N}} .
$$

This indicates that gravitational cutoff in a theory with $N$ species cannot be higher than $p^{*}$.

This result reconciles the bound on the Planck mass with naturalness arguments. The perturbative renormalization of the Planck mass due to the species is given by the quadratically divergent part of the two point function of $T$. In absence of other contributions and assuming no cancellations, the net contribution to the Planck mass is expected to be

$$
M_{P}^{2} \approx N \Lambda_{G}^{2}
$$

with $\Lambda_{G}$ the cutoff, which reproduces Eq. (3.7).

Let us mention that the lowering of the cutoff due to the large number of species resembles the situation in gauge theories with positive $\beta$-function. Consider, for example, a $U(1)$ gauge theory with $N$ flavor species. Above the mass of the heaviest particle, the one loop correction to the gauge coupling is given by

$$
g(p)^{2}=g(\mu)^{2}+N \frac{g(\mu)^{4}}{12 \pi^{2}} \log \frac{p^{2}}{\mu^{2}}
$$

which increases the gauge coupling at short distances. The perturbative expansion goes out of control at the scale

$$
p^{*}=\mu e^{\left(12 \pi^{2}\right) /\left(g(\mu)^{2} N\right)} .
$$

This of course corresponds to the Landau pole of the theory below which the effective theory must break down. In order for the effective theory to have a minimal regime of validity, clearly one should have

$$
g(\mu)^{2} N \lesssim 12 \pi^{2}
$$

so that there is a separation between the Landau pole and $\mu$. For a given $g(\mu)$ the cutoff gets reduced (exponentially) as we increase the number of species. We should here note however that in the gauge theory case with a large number of colors, the correction has the opposite sign so that the coupling is reduced at short distances and the corrections can be resummed. It would interesting to see if there exists an analog in the gravity case. A hint that this must be the case is given by the fact that, while in string theory there exists an infinite tower of states, nevertheless the graviton amplitudes are manifestly soft at high energy. For ordinary matter with spin less or equal than one, it follows from unitarity that the sign of the correction always makes 
gravity stronger at short distances because the central charge must be positive. One possibility is that this might be violated in theories containing higher spins such as string theory. We leave this question for future work.

\section{Maximal temperature}

We would like now to show that the existence of the scale $\Lambda_{G}$ can also be inferred by considering the thermodynamics of the $N$ species. The basic idea that we want to present is that a theory with $N$ species has a limiting temperature set by $M_{P} / \sqrt{N}$, or else it will be possible to prepare systems which violate Bekenstein's entropy bound [6]. The connection between maximal temperature and entropy bounds was also considered in [7]. It would be also interesting to explore the connection with other entropy bounds [8].

Let us consider the following gedanken experiment, depicted in Fig. 2. Take a box of size $L$ in which all the species are heated at temperature $T$ much above their mass $\Lambda$ so that the particles can be treated as relativistic. Under the assumption that thermal distribution for all the species can be reached, the energy stored in the box is given by ${ }^{1}$

$$
E \approx N T^{4} L^{3}
$$

The size $L$ of the box is limited by the fact that the system will eventually collapse into a black hole. Working in approximately flat space, i.e. neglecting gravity backreaction, is justified if the Schwarzchild radius of the black hole with the same energy is smaller than $L$. This gives the upper bound on the size of the system,

$$
L_{\max } \approx \frac{M_{P}}{T^{2} \sqrt{N}}
$$

We can now easily find a constraint on $T$. The entropy of the gas of $N$ relativistic species is

$$
S_{\mathrm{gas}} \approx N T^{3} L^{3} \text {. }
$$

This entropy should not exceed the Bekenstein's bound [6],

$$
S \leq S_{\mathrm{Bek}} \approx M_{P}^{2} L^{2} .
$$

By taking a box of maximal size (3.13), one finds that for this system there exists a maximal temperature given by

$$
T_{\max } \lesssim \frac{M_{P}}{\sqrt{N}}
$$

i.e. the particles cannot be heated above $\Lambda_{G}$. In particular, if the hierarchy problem is explained by the large $N$ number of species $\Lambda_{G} \approx \Lambda$, so that it is impossible to

\footnotetext{
${ }^{1}$ Clearly because of weak interspecies interactions, particles may only thermalize within their own species or not thermalize at all. However, what is needed for the argument is that they have a long enough maintained thermal distribution. This would be the case, for example, if the particles are produced by GibbonsHawking radiation in a time dependent background.
}

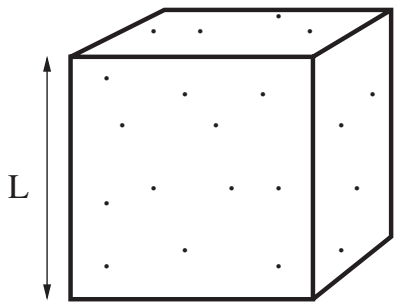

FIG. 2. A box filled with radiation violates the entropy bound prematurely.

heat all the species at the temperature above their mass. It is natural to interpret the existence of this maximal temperature as a cutoff. In fact in theories with a small number of species this becomes simply the statement that $M_{P}$ is the limiting temperature.

As an example, let us consider the large extradimensions case [4]. In this context the previous bound can be understood as follows. If we consider the higher dimensional theory with fundamental Planck scale $M_{D}$ of order $\mathrm{TeV}$, this is obviously the maximum temperature allowed for the system before compactification. In the experiment considered above at high temperature, the size of the box (3.13) is much smaller that the size of the extra dimension so that effectively we are probing the theory in the decompactified limit. From the lower dimensional point of view, the limiting temperature is simply understood as due to the collective effect of the tower of 4D Kaluza-Klein (KK) states. We would like to stress however that this bound is derived in a model independent way and relies only on the assumption that the system has a fixed number of degrees of freedom.

It is interesting to note that an alternative derivation of the maximal temperature can be obtained considering the following simple cosmological setup. ${ }^{2}$ Take an FRW universe filled with $N$ species of mass $\Lambda$ or below whose temperature $\Lambda \ll T \ll \Lambda_{G}$. Because the temperature is below the quantum gravity scale, and species are relativistic, such Universe will expand as a radiation dominated FRW Universe with Hubble parameter,

$$
H \approx \frac{\sqrt{N} T^{2}}{M_{P}} \approx \frac{T^{2}}{\Lambda} .
$$

The first immediate upper bound on $T$ comes from the requirement that Hubble be sub-Planckian, which gives $T \approx \sqrt{M_{P} \Lambda}$. However, a much more stringent bound arises from noticing that the temperature of the relativistic fluid becomes smaller than the horizon temperature $H$ at $T_{\max }$ given by Eq. (3.16). This should be clearly impossible

\footnotetext{
${ }^{2}$ This approach relies on the assumption that the standard 4D Freedman-Robertson-Walker (FRW) cosmology is applicable up to the temperature of interest. In particular, this is not the case in the large extra-dimension scenario where gravity becomes higher dimensional at much lower bulk temperature.
} 
since the quantum fluctuations of the background will automatically increase the temperature up to $H$.

\section{EXAMPLES}

In this section we consider examples where the bound (1.1) is saturated.

First, let us note that the black hole bound sheds a very different light on the large extra-dimension solution to the hierarchy problem. Here the weakness of gravity is accounted by the large volume of the extra dimensional space, which dilutes gravity and sets the hierarchy between $M_{P}$ and the fundamental gravity scale $\Lambda_{G}$,

$$
M_{P}^{2}=\Lambda_{G}^{2}\left(\Lambda_{G} R\right)^{n},
$$

where $R$ is the typical size of the extra dimensions, and $n$ is their number. This relation acquires a very different meaning if we note that the volume of the extra space determines the number of KK species with mass $\Lambda_{G}$ in the following way:

$$
\left(\Lambda_{G} R\right)^{n}=N
$$

With this observation the relation between the fourdimensional and high-dimensional Planck masses is nothing but the saturation of the bound (1.1) [or equivalently (1.2)]. Looking from this point of view, the large extradimensions model solves the hierarchy problem due to the fact that it includes $\approx 10^{32} \mathrm{KK}$ gravitons of mass less than $\mathrm{TeV}$.

Hence, the large extra-dimension scenario can be regarded as a particular example of a much larger class of models in which the hierarchy problem is solved due to a large number of species. Having $\approx 10^{32}$ copies of the SM is another example from this class, which has some advantages with respect to higher dimensional geometric picture, since one does not have to worry about the stabilization of the radius modulus. In addition, the cosmological constraints become less severe, due to the fact that, unlike the production rate of KK gravitons, the production of other species is very strongly suppressed.

Another interesting example is provided by the RandallSundrum (RS) scenario [9]. We focus for simplicity on the model with one flat brane embedded in $\mathrm{AdS}_{5}$. Although this model does not solve the hierarchy problem due to the number of species, nevertheless it saturates the bound on the Planck mass and the effective number of degrees of freedom of the theory. From the 4D point of view, the model is described by a normalizable massless $4 \mathrm{D}$ graviton and a continuum of KK modes. The 4D Planck mass is given by

$$
M_{P}^{2}=\frac{M_{5}^{3}}{k},
$$

where $k$ is the $\mathrm{AdS}_{5}$ curvature and $M_{5}$ the 5D Planck scale. The physics of this model is greatly clarified by turning to the holographic description. Using the AdS/CFT corre- spondence [10], RS is dual to a 4D strongly coupled "large $N$ " conformal field theory (CFT) coupled to gravity [11]. The CFT is broken in the ultraviolet at a scale $k$ and has a central charge related given by $c=M_{5}^{3} / k^{3}$. While the CFT does not have particles in the usual sense, given that the central charge measures the effective number of degrees of freedom of the theory, one sees that Eq. (4.3) can be written as

$$
M_{P}^{2} \approx N k^{2}
$$

which saturates the bound (1.1) since the broken CFT has excitations up to energy $k$.

It is important to clarify the nature of the cutoff in this scenario. The description of RS in terms of a CFT coupled to gravity is valid up to the scale $k$ where the CFT is broken. A possible UV completion of the broken CFT is precisely given by the 5D geometric setup. At energies larger than $k$, the AdS curvature becomes irrelevant and the physics turns higher dimensional. The breakdown of the perturbative expansion is then simply understood as the transition to the five-dimensional regime. However, in this case gravity does not become strongly coupled until the fundamental Planck scale $M_{5}$ which one can take arbitrarily larger than $k$.

More speculatively, it is interesting to note that the bound between the Planck scale and the number of species might be saturated in perturbative string theory [12]. Generalizing the argument of [1], one finds that in arbitrary dimensions

$$
M_{D}^{D-2} \gtrsim N \Lambda^{D-2} .
$$

This is because the temperature of a $\mathrm{BH}$ of mass $M_{\mathrm{BH}}$ in $D$ dimensions is given by $T^{D-3}=M_{D}^{D-2} / M_{B H}$. From this it follows that the $\mathrm{BH}$ can at most emit $M_{D}^{D-2} / \Lambda^{D-2}$ particles of mass $\Lambda$. If there are $N$ species with approximately conserved species number to avoid inconsistencies, one finds that the Planck mass has to be scaled as in (4.5). Similarly the other arguments presented in Sec. III generalize to arbitrary dimension by replacing $M_{P}^{2}$ with $M_{D}^{D-2}$ and scaling the other dimensionful quantities with the appropriate power.

Let us now consider critical string theory in 10D. Here one finds

$$
M_{10}^{8} \approx \frac{m_{s}^{8}}{g^{2}},
$$

where $m_{s}$ is the string scale and $g$ the string coupling. It is tempting to interpret $1 / g^{2}$ as the effective number of degrees of freedom so that the $M_{10}$ assumes the natural value. Note that this is much less than the number of string modes between the $M_{10}$ and $m_{s}$ which grows exponentially. The reduction of number of degrees of freedom has also been conjectured by Atick and Witten [13] in relation to the Hagedorn phase transition. The existence of a limit- 
ing temperature $T_{\mathrm{Hag}} \approx m_{s}$ in fact also agrees with the temperature argument presented above.

\section{STRONG GRAVITY AT $\Lambda_{G} \approx \mathrm{TeV}$}

In the previous sections we have shown that in a theory with $N$ species there exists an effective cutoff in the gravity sector given by $\Lambda_{G} \approx M_{P} / \sqrt{N}$. One natural possibility is that $\Lambda_{G}$ is the quantum gravity scale. We focus here on model independent properties of black holes in the scenarios with many species solution to the hierarchy problem in which gravity becomes strong at $\Lambda_{G}$.

Since we assume that gravity becomes strong at the scale $\Lambda_{G} \approx \Lambda$, one will start probing quantum gravity in high energy particle collisions at the $\mathrm{TeV}$ scale. In particular, one should observe a significant production of microscopic BHs at LHC. At first sight, this production leads us to a puzzle, the resolution of which provides interesting information about the effective $\mathrm{BH}$ interactions in such theories.

Consider a process with a characteristic energy $E$, in which a particle-antiparticle pair of the $j$ th specie, is produced in the annihilation of the two SM states (specie $i)$, via the virtual $\mathrm{BH}$ exchange,

$$
\psi_{i}+\bar{\psi}_{i} \rightarrow B H \rightarrow \psi_{j}+\bar{\psi}_{j} .
$$

The rate of the process goes as

$$
\Gamma_{i \rightarrow j} \approx \frac{E^{9}}{M_{i}^{2} M_{j}^{2} M_{\mathrm{BH}}^{4}},
$$

where $M_{i}$ are the mass scales controlling the effective BH coupling to the SM and to the other species.

Let us assume that $M_{i}$ are universally of order of some typical scale $M$. To establish the bound on the scale $M$, we consider the total production rate in which all possible $j$ species are produced in the annihilation of the SM particles. Assuming that the partial rates are $j$-independent, the result is obtained by simple multiplication of (5.2) by $N \approx$ $M_{P}^{2} / \Lambda^{2}$

$$
\Gamma_{\mathrm{TOTAL}}=\sum_{j}^{N} \Gamma_{i \rightarrow i} \approx \frac{E^{9}}{\Lambda^{8}}\left(\frac{M_{P}^{2} \Lambda^{2}}{M^{4}}\right),
$$

where we have used the fact that $M_{\mathrm{BH}} \approx \Lambda$. Requiring that the above rate does not violate unitarity at least until the energy $E \approx \Lambda$, we get the bound

$$
M \gtrsim \sqrt{M_{P} \Lambda} .
$$

In other words, if we assume universality of the couplings, unitarity implies that the probability of production must be small in conflict with the assumption of gravity becoming strong at $\Lambda$.

An even more severe lower bound on $M$ can be derived from the following argument. Because the intermediate $\mathrm{BH}$ is heavy, below the scale $\Lambda$ it can be integrated out. Then, the same diagram that was generating the interspecies scattering process (5.1) generates an effective higher dimensional operator of the form

$$
\frac{T_{\mu \nu}^{(i)} T^{\mu \nu(j)}}{M_{i} M_{j} M_{\mathrm{BH}}^{2}}+\cdots,
$$

where $T_{\mu \nu}^{(i)}$ is the energy momentum tensors of the $i$ th specie. Other structures compatible with the symmetries, e.g., coupling between the vector or scaler currents, are of course also possible.

Since below the scale $\Lambda$, the above interaction can be treated as a fundamental vertex, it will generate quantum corrections to the Lagrangian of the SM fields. In particular, there will be a significant renormalization of the kinetic terms of the SM fields from $\psi_{j}$ loops, due to their enormous multiplicity. Putting aside accidental cancellations, the wave function renormalization factor of the SM fields will scale as

$$
Z_{\text {eff }} \gtrsim \frac{M_{P}^{2}}{M^{2}} .
$$

Requiring these effects to be small implies

$$
M \gtrsim M_{P} \text {. }
$$

Hence, the interspecies production rate through the $\mathrm{BH}$ exchange would be as suppressed as the one mediated by graviton itself.

The inevitable conclusion is that either BHs should have suppressed effective couplings to all the species or the assumption about the universality of this coupling is wrong. The former option would be inconsistent with gravity becoming strong at the scale $\Lambda$. Thus, we are lead to the conclusion that $\mathrm{BH}$ coupling cannot be universal and somehow must carry a label that allows them to differentiate among the species, and predominantly decay into the specie of origin.

Indeed, it is easy to see that the latter possibility is compatible with the notion of strong gravity at scale $\Lambda$ and makes the whole picture consistent. Relaxing the assumption of universality of the scale $M_{j}$, the total rate of production of species via $\mathrm{BH}$ exchange in the collision of given $i$ th species now becomes

$$
\Gamma_{\mathrm{TOTAL}}^{(i)}=\sum_{j} \Gamma_{i \rightarrow j} \approx \frac{1}{M_{i}^{2}} \frac{E^{9}}{\Lambda^{4}}\left(\sum_{j}^{N} \frac{1}{M_{j}^{2}}\right),
$$

which puts a constraint

$$
\frac{1}{M_{i}^{2}}\left(\sum_{j}^{N} \frac{1}{M_{j}^{2}}\right) \lesssim \frac{1}{\Lambda^{4}} .
$$

Similarly the renormalization of the SM kinetic terms now gives the bound

$$
\frac{1}{M_{i}}\left(\sum_{j}^{N} \frac{1}{M_{j}}\right) \lessgtr \frac{1}{\Lambda^{2}} .
$$

Both bounds are automatically satisfied and reconciled 
with gravity becoming strong at the scale $\Lambda$, if we assume that the $\mathrm{BH}$ produced in the collision of $i$ th species decay to the same species, with the rate $\approx \Lambda$, whereas the decay rate into the other species is suppressed by $\approx \Lambda / M_{P}^{2}$.

This means that micro-BHs admit a certain notion of locality in the space of species. The locality implies that BHs are endowed with a hair, that gives them a memory of their site of origin in the space of species. This hair may not necessarily be permanent, but it has to last long enough in order to suppress the decay rate into other species. In such a case, the hair will decay after certain characteristic time, and the memory about the origin will fade away, allowing decay into foreign species.

It is important to note that existence of such a hair is not in any conflict with the standard classical BH no-hair theorems [14]. First, the hair that establishes the connection between the $\mathrm{BH}$ and the site of origin can be quantum mechanical and does not need to be static. It was discovered by Krauss and Wilczek some time ago [5] that BH can carry a quantum-mechanical hair under discrete gauge $Z_{N}$ symmetries. More recently, it was pointed out [15] that, in the presence of spin-2 or higher integer-spin massive fields, the black holes may be endowed with the quantum hair under these fields. Moreover, the BHs themselves are not classical BHs in the sense of four-dimensional gravity, since their gravitational radius is within their Compton wavelength. Therefore, although the BHs may appear classical from the point of view of an underlying classical theory that operates at intermediate distances larger than $\Lambda^{-1}$, they certainly are not classical in the usual sense. They only become classical from the point of view of 4D gravity when their mass reaches $M_{P}$, in which case universality of the decay is restored.

To clarify the above statements we turn once more to the large extra-dimension scenario. There the relevant scales are the radius of the extra dimensions $R$, the mass of a black hole $M_{\mathrm{BH}}$, the fundamental quantum gravity scale $\Lambda_{G}$, and the (four-dimensional) Planck mass $M_{P}$. BHs of intermediate size $\Lambda_{G} \ll M_{\mathrm{BH}} \ll M_{P} R^{2}$ have a size larger than $1 / \Lambda_{G}$, but smaller than the size of the extra dimension $R$. While such BHs can be regarded as classical from the point of view of the high-dimensional classical gravity operating and distances $\ll R$, in no sense such objects are standard BHs from the point of view of a fourdimensional low energy observer, probing gravity with the zero mode graviton. Therefore, neither four nor higher dimensional observers would be surprised to discover that such BHs are not universally coupled. Indeed, they are not. A micro-BH produced in a collision on a given brane will mostly decay into the species localized on the brane of origin and only with very suppressed probability into states localized at some $\approx R$ distance away. Only the very heavy BHs, with masses $\gg M_{P}^{2} R$, can reach across the bulk and start decay universally. This of course agrees with the fact that these BHs are classical already in the 4D description and therefore universality is guaranteed by classical $\mathrm{BH}$ theorems.

To conclude, while the simplest way to visualize locality is to imagine the species separated in some physical space, what we are finding is that locality in the space of species is something more general, since at no point in our discussion we have assumed the existence of underlying extra dimensions, in which the species are separated.

\section{ACKNOWLEDGMENTS}

We thank R. Brustein, G. Gabadadze, O. Nielsen, O. Pujolas, R. Rattazzi, and G. Veneziano for very useful discussions and comments. The work of G.D. and M. R. is supported in part by David and Lucile Packard Foundation Fellowship for Science and Engineering, and by NSF Grant No. PHY-0245068.
[1] G. Dvali, arXiv:0706.2050.

[2] G. R. Dvali and G. Gabadadze, Phys. Rev. D 63, 065007 (2001); G. R. Dvali, G. Gabadadze, M. Kolanovic, and F. Nitti, Phys. Rev. D 65, 024031 (2001).

[3] G. Veneziano, J. High Energy Phys. 06 (2002) 051.

[4] N. Arkani-Hamed, S. Dimopoulos, and G. R. Dvali, Phys. Lett. B 429, 263 (1998); Phys. Rev. D 59, 086004 (1999); I. Antoniadis, N. Arkani-Hamed, S. Dimopoulos, and G. R. Dvali, Phys. Lett. B 436, 257 (1998).

[5] L. M. Krauss and F. Wilczek, Phys. Rev. Lett. 62, 1221 (1989); J. Preskill and L. M. Krauss, Nucl. Phys. B341, 50 (1990); S. Coleman, J. Preskill, and F. Wilczek, Phys. Rev. Lett. 67, 1975 (1991).

[6] J. D. Bekenstein, Phys. Rev. D 23, 287 (1981); 49, 1912 (1994).
[7] R. Brustein and G. Veneziano, Phys. Rev. Lett. 84, 5695 (2000); R. Brustein, S. Foffa, and G. Veneziano, Phys. Lett. B 507, 270 (2001).

[8] W. Fischler and L. Susskind, arXiv:hep-th/9806039; R. Bousso, J. High Energy Phys. 07 (1999) 004.

[9] L. Randall and R. Sundrum, Phys. Rev. Lett. 83, 3370 (1999); 83, 4690 (1999).

[10] J. M. Maldacena, Adv. Theor. Math. Phys. 2, 231 (1998); Int. J. Theor. Phys. 38, 1113 (1999); S. S. Gubser, I. R. Klebanov, and A. M. Polyakov, Phys. Lett. B 428, 105 (1998); E. Witten, Adv. Theor. Math. Phys. 2, 253 (1998).

[11] N. Arkani-Hamed, M. Porrati, and L. Randall, J. High Energy Phys. 08 (2001) 017; R. Rattazzi and A. Zaffaroni, J. High Energy Phys. 04 (2001) 021.

[12] We understand that this has also been conjectured by $\mathrm{G}$. 
Veneziano.

[13] J. J. Atick and E. Witten, Nucl. Phys. B310, 291 (1988).

[14] J. Bekenstein, Phys. Rev. D 5, 1239 (1972); 5, 2403 (1972); Phys. Rev. Lett. 28, 452 (1972); C. Teitelboim, Phys. Rev. D 5, 2941 (1972); W. Israel, Phys. Rev. 164,
1776 (1967); Commun. Math. Phys. 8, 245 (1968); B. Carter, Phys. Rev. Lett. 26, 331 (1971); J. Hartle, Phys. Rev. D 3, 2938 (1971).

[15] G. Dvali, Phys. Rev. D 74, 044013 (2006); arXiv:hep-th/ 0607144. 